\title{
Impulsive Control and Synchronization of a New 5D Hyperchaotic System
}

\author{
K. Kemin ${ }^{a}$, H. Bouraoui ${ }^{a}$, M. Messadi ${ }^{a}$ And M. GHAnes ${ }^{b}$ \\ ${ }^{a}$ EACSys-Lamel Laboratory, Jijel University, Jijel, Algeria \\ ${ }^{b}$ ECS-ENSEA, 06 av. du Ponceau, 95014 Cergy-Pontoise Cedex, France
}

\begin{abstract}
This paper investigates the control and the synchronization of a new 5D hyperchaotic system. Based on the impulsive control theory, some new and less conservative criteria for the global exponential stability and asymptotical stability of impulsively controlled 5D hyperchaotic system, are obtained with varying impulsive interval. Finally, numerical simulations are given to demonstrate the effectiveness of the proposed control and synchronization methodology.
\end{abstract}

DOI: $10.12693 /$ APhysPolA.123.193

PACS: 05.45.Gg, 05.45.Xt

\section{Introduction}

Recently, hyperchaotic systems have begun to attract considerable attention due to their theoretical and practical applications in lasers [1], mechanical engineering [2], and secure communication [3], and so on. Controlling these complex dynamics for engineering applications has emerged as a new and attractive field and has developed many profound theories and methodologies $[4,5]$.

In this paper, we study the control towards an equilibrium point by impulsive control method of the 5th-order hyperchaotic system given by [6]:

$$
\begin{aligned}
& \dot{x}_{1}=a_{1}\left(x_{2}-x_{1}\right)+x_{2} x_{3} x_{4} x_{5}, \\
& \dot{x}_{2}=a_{2}\left(x_{1}+x_{2}\right)-x_{1} x_{3} x_{4} x_{5}, \\
& \dot{x}_{3}=-x_{3}+0.1 x_{1}^{2}, \\
& \dot{x}_{4}=-a_{3} x_{4}+x_{1} x_{2} x_{3} x_{5}, \\
& \dot{x}_{5}=-a_{4}\left(x_{5}-x_{4}\right)-a_{5} x_{1}+x_{1} x_{2} x_{3} x_{4},
\end{aligned}
$$

where $x_{1}, x_{2}, x_{3}, x_{4}$, and $x_{5}$ are state variables, $a_{1}, a_{2}$, $a_{3}, a_{4}$, and $a_{5}$ are all positive real parameters. When we selected the parameters as $a_{1}=37, a_{2}=14.5, a_{3}=10.5$, $a_{4}=15$, and $a_{5}=9.5$, the system exhibits a hyper-chaotic behaviour.

\section{Impulsive control of the 5D hyperchaotic system}

We decompose the linear and nonlinear parts of the 5D hyperchaotic system in Eq. (1) and rewrite it as

$$
\dot{x}=A x+\Phi(x),
$$

where

$$
\begin{aligned}
& x=\left[x_{1} x_{2} x_{3} x_{4} x_{5}\right]^{\mathrm{T}}, \\
& A=\left|\begin{array}{ccccc}
-a_{1} & a_{1} & 0 & 0 & 0 \\
a_{2} & a_{2} & 0 & 0 & 0 \\
0 & 0 & -1 & 0 & 0 \\
0 & 0 & 0 & -a_{3} & 0 \\
a_{5} & 0 & 0 & a_{4} & -a_{5}
\end{array}\right|
\end{aligned}
$$

and

$$
\Phi(x)=\left[\begin{array}{c}
x_{2} x_{3} x_{4} x_{5} \\
-x_{1} x_{3} x_{4} x_{5} \\
0.1 x_{1}^{2} \\
x_{1} x_{2} x_{3} x_{5} \\
x_{1} x_{2} x_{3} x_{4}
\end{array}\right] .
$$

Then, the impulsive control of the critical system is given by $(6)$ :

$$
\left\{\begin{array}{l}
\dot{x}=A x+\Phi(x), \quad t \neq t_{i}, \\
\Delta x=B_{i} x, \quad t=t_{i}, \quad i=1,2, \ldots, \\
x\left(t_{0}^{+}\right)=x_{0},
\end{array}\right.
$$

where $t_{i}$ denotes the instant when impulsive control occurs. For convenience, define the following notation:

$$
\begin{aligned}
& \tilde{\lambda}(A)=\frac{1}{2} \lambda_{\max }\left(A+A^{\mathrm{T}}\right), \\
& \beta_{i}=\lambda_{\max }\left[\left(I+B_{i}\right)^{\mathrm{T}}\left(I+B_{i}\right)\right],
\end{aligned}
$$

where $I$ is the identity matrix, and $\lambda_{\max }(A)$ is the maximal eigenvalue of matrices $A$.

Theorem 1 [7]:

- The trivial solution of the system (5) is globally exponentially stable if $\tilde{\lambda}(A)=\frac{\lambda}{2}<0$ and there exists a constant $0 \leq \alpha<-\lambda$, such that $\ln \beta_{i}-$ $\alpha\left(t_{i}-t_{i-1}\right) \leq 0, i=1,2, \ldots$

- The trivial solution of the system (5) is globally asymptotically stable if $\tilde{\lambda}(A)=\frac{\lambda}{2} \geq 0$ and there exists a constant $\alpha>1$, such that $\ln \left(\alpha \beta_{i}\right)+$ $\lambda\left(t_{i+1}-t_{i}\right) \leq 0, i=1,2, \ldots$

\section{Impulsive synchronization of the $5 \mathrm{D}$ hyperchaotic system}

In this section, we will study the impulsive synchronization of two identical hyperchaotic systems. Let system (5) be the drive system, and the response system is 
modeled by the following impulsive equation:

$$
\left\{\begin{array}{l}
\dot{y}=A y+\Phi(y), \quad t \neq t_{i}, \\
\Delta y=B_{i} y, \quad t=t_{i}, \quad i=1,2, \ldots, \\
y\left(t_{0}^{+}\right)=y_{0} .
\end{array}\right.
$$

Consider the error vector

$$
e=y-x=A e+\Phi(y)-\Phi(x) .
$$

Then the error system of the impulsive synchronization is given by

$$
\left\{\begin{array}{l}
\dot{e}=A e+\Phi(y)-\Phi(x), \quad t \neq t_{i}, \\
\Delta e=B_{i} e, \quad t=t_{i}, \quad i=1,2, \ldots, \\
e\left(t_{0}^{+}\right)=y_{0}-x_{0} .
\end{array}\right.
$$

Similarly to the stabilization of the hyperchaotic system, the value of $\alpha$ was calculated using theorem 1 .

\section{Numerical simulation}

In this section, numerical simulations are given to verify the effectiveness of the impulsive control and synchronization of hyperchaotic 5D system. The fourth-order Runge-Kutta integration method is used to solve the system with time step size equal to 0.0001 , the initial values are $[2,-2,3.5,-3.5,5]$.

We start by calculating the matrix $A+A^{\mathrm{T}}=$ $\left[\begin{array}{cccc}-20 & 50 & 0 & -10.6 \\ 50 & 0 & 0 & 1 \\ 0 & 0 & -5 & 0 \\ -10.6 & 1 & 0 & 0\end{array}\right]$

eigenvalues, which are: $96.35,-23.20,-2.00,26.01$, and 50.55. Then, the maximal eigenvalue is $\tilde{\lambda}(A)=$ $50.55>0$. If we choose the gain matrices $B_{i},(i=$ $1,2, \ldots)$ as a constant matrix $B=\operatorname{diag}\left(b_{1}, b_{2}, b_{3}, b_{4}, b_{5}\right)=$ $(-0.95,-0.95,-0.95,-0.95,-0.95)$, then it is easy to see that: $\beta=\max \left(\left(1+b_{1}\right)^{2},\left(1+b_{2}\right)^{2},\left(1+b_{3}\right)^{2},\left(1+b_{4}\right)^{2}\right.$, $\left.\left(1+b_{5}\right)^{2}\right)=0.0025$. The estimates of bounds of stable regions are given by $0 \leq \tau \leq-\frac{\ln \alpha+\ln (0.0025)}{50.55}$. If we choose $\alpha=2$, then $0 \leq \tau \leq 0.15$.
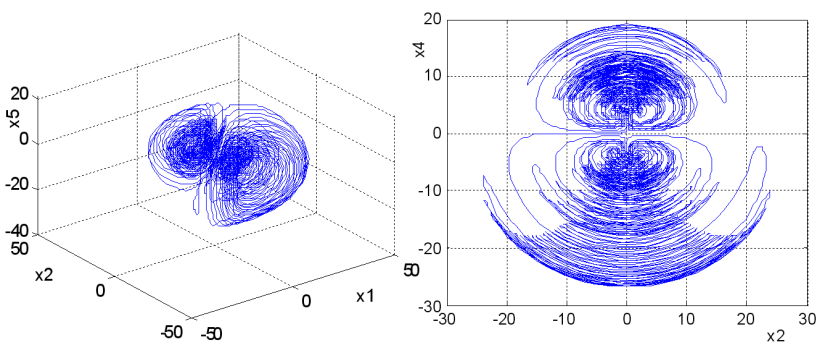

Fig. 1. The hyperchaotic attractor obtained by integrating numerically the system (1).

Figure 1 shows the attractor of the 5D hyperchaotic system, Fig. 2 shows the impulsive control with $\tau=0.10 \mathrm{~s}$ of the 5D hyperchaotic system to origin when control is activated at $t=2.5 \mathrm{~s}$. As can be seen, the system stabilizes after $0.5 \mathrm{~s}$. If the impulse intervals are too

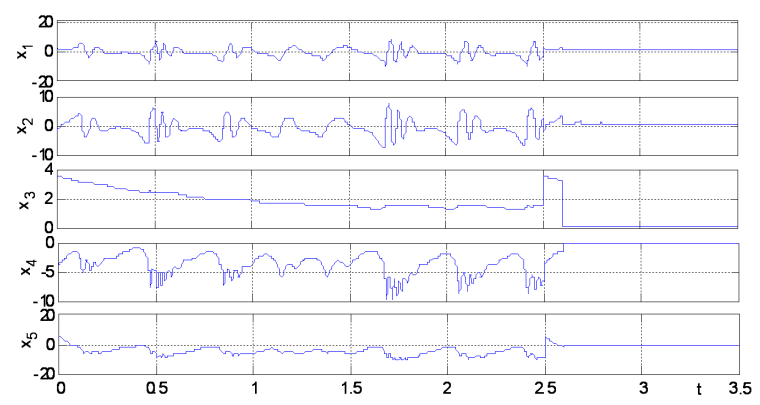

Fig. 2. Impulsive control with $\tau=0.10 \mathrm{~s}$ of the new hyperchaotic system to origin when control is activated at $t=2.5 \mathrm{~s}$.

large $\tau>0.3$, the impulsively controlled system cannot be stabilized, as shown in Fig. 3 with $\tau=0.6$.

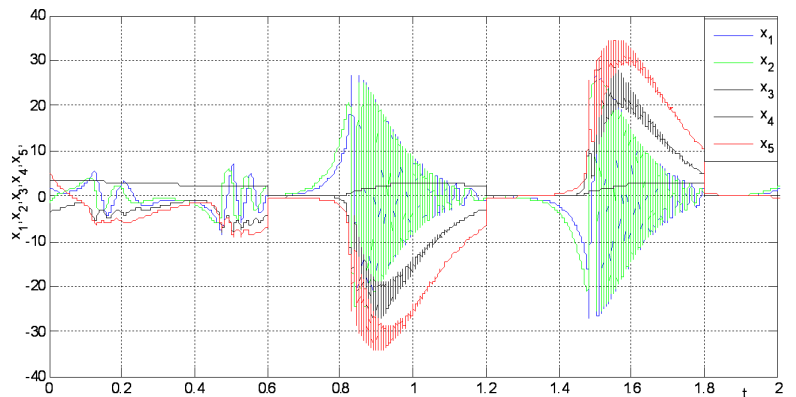

Fig. 3. Impulsive control with $\tau=0.6 \mathrm{~s}$ of the new hyperchaotic system to origin.

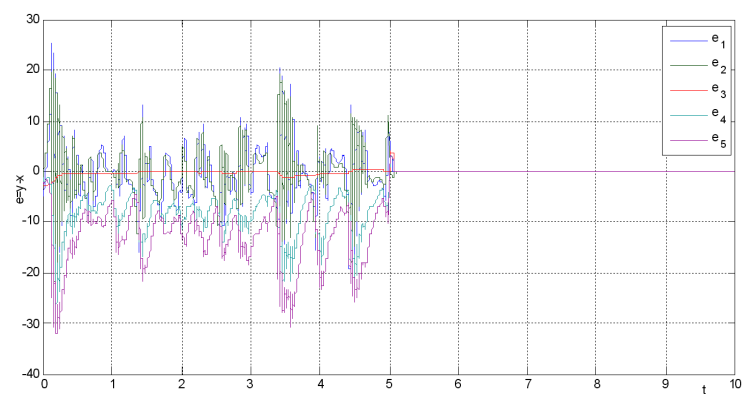

Fig. 4. Synchronization errors of two 5D hyperchaotic systems with $\tau=0.05$ when control is activated at $t=5 \mathrm{~s}$.

Figure 4 shows the results when $\tau=0.05$. It is clear that with this impulsive control, two identical 5D hyperchaotic systems synchronize very fast.

\section{Conclusion}

This paper has studied the impulsive control and synchronisation of 5D hyperchaotic system. Some new and less conservative criteria for the global exponential stability and asymptotical stability of impulsively controlled 
hyperchaotic system are obtained with varying the impulsive intervals. The performances of the proposed approach have been verified by the numerical simulations.

\section{References}

[1] V. Ahlers, U. Parlitz, W. Lauterborn, Phys. Rev. E 58, 7208 (1998)

[2] Y.X. Luo, D.X. Li, X. Wu, J. Mech. Eng. 54, 372 (2008).

[3] X.J. Wu, H. Wang, H.T. Lu, Nonlinear Anal., Real World Appl. 12, 1288 (2011)
[4] Y.X. Li, G.R. Chen, W.K.S. Tang, IEEE Trans Circuits Syst. II 52, 204 (2005).

[5] S. Bououden, S. Filali, K. Karim, Int. J. Innov. Comput. I Control 6, 541 (2010).

[6] K. Kemih, H. Bouraoui, M. Ghanes, R. Remmouche, A. Senouci, Int. J. Modelling, Identification Control 17, 206 (2012).

[7] T. Yang, Impulsive Control Theory, Springer-Verlag, Berlin 2001. 